\title{
Distribution of some quadratic linear recurrence sequences modulo 1
}

\section{ARTŪRAS DUBICKAS}

\begin{abstract}
.
We show that if $a$ is an even integer then for every $\xi \in \mathbb{R}$ the smallest limit point of the sequence $\left\|\xi a^{n}\right\|_{n=1}^{\infty}$ does not exceed $|a| /(2|a|+2)$ and this bound is best possible in the sense that for some $\xi$ this constant cannot be improved. Similar (best possible) bound is also obtained for the smallest limit point of the sequence $\left\|\xi x_{n}\right\|_{n=1}^{\infty}$, where $\left(x_{n}\right)_{n=1}^{\infty}$ satisfies the second order linear recurrence $x_{n}=a x_{n-1}+b x_{n-2}$ with $a, b \in \mathbb{N}$ satisfying $a \geqslant b$. For the Fibonacci sequence $\left(F_{n}\right)_{n=1}^{\infty}$ our result implies that $\sup _{\xi \in \mathbb{R}} \liminf _{n \rightarrow \infty}\left\|\xi F_{n}\right\|=$ $1 / 5$, and e.g., in case when $a \geqslant 3$ is an odd integer, $b=1$ and $\theta:=a / 2+\sqrt{a^{2} / 4+1}$ it shows that $\sup _{\xi \in \mathbb{R}} \liminf \operatorname{in}_{n \rightarrow \infty}\left\|\xi \theta^{n}\right\|=(a-1) / 2 a$.
\end{abstract}

Acknowledgment. I thank the referee for a useful suggestion.

\section{REFERENCES}

[1] Akhunzhanov, R. K. and Moshchevitin, N. G., On the chromatic number of a distance graph associated with a lacunary sequence, Dokl. Ross. Akad. Nauk, 397 (2004), 295-296 (in Russian)

[2] Bugeaud, Y., Distribution modulo one and Diophantine approximation, Cambridge University Press, 2012

[3] Carroll, D., Jacobson, E. and Somer, L., Distribution of two-term recurrence sequences mod pe , Fibonacci Q., 32 (1994), 260-265

[4] Cassels, J. W. S., An introduction to Diophantine approximation, Cambridge University Press, 1957

[5] de Mathan, B., Numbers contravening a condition in density modulo 1, Acta Math. Acad. Sci. Hung., 36 (1980), 237-241

[6] Dubickas, A., Arithmetical properties of powers of algebraic numbers, Bull. London Math. Soc., 38 (2006), 70-80

[7] Dubickas, A., On the fractional parts of lacunary sequences, Math. Scand., 99 (2006), 136-146

[8] Dubickas, A., On the distance from a rational power to the nearest integer, J. Number Theory, 117 (2006), 222-239

[9] Dubickas, A., On the limit points of the fractional parts of powers of Pisot numbers, Archivum Mathematicum, 42 (2006), 151-158

[10] Erdős, P., Problems and results on Diophantine approximations. II, Repartition modulo 1, Actes Colloq. Marseille-Luminy 1974, Lecture Notes in Math., 475 (1975), 89-99

[11] Kaneko, H., Distribution of geometric sequences modulo 1, Result. Math., 52 (2008), 91-109

[12] Kaneko, H., Limit points of fractional parts of geometric sequences, Unif. Distrib. Theory, 4 (2) (2009), 1-37

[13] Katznelson, Y., Chromatic numbers of Cayley graphs on $\mathbb{Z}$ and recurrence, Combinatorica, 21 (2001), 211-219

[14] Khintchine, A., Über eine Klasse linearer diophantischer Approximationen, Rend. Circ. Mat. Palermo, 50 (1926), 170-195

[15] Moshchevitin, N. G., Density modulo 1 of lacunary and sublacunary sequences: application of Peres-Schlag's construction, Journal of Mathematical Sciences, 180 (2012), 610-625

[16] Peres, Y. and Schlag, W., Two Erdős problems on lacunary sequences: chromatic number and Diophantine approximation, Bull. London Math. Soc., 42 (2010), 295-300

[17] Pollington, A. D., On the density of the sequence $\left\{n_{k} \xi\right\}$, Illinois J. Math., 23 (1979), 511-515

[18] Schinzel, A., Special Lucas sequences, including the Fibonacci sequence, modulo a prime, A tribute to Paul Erdós (A. Baker et all, eds.), Cambridge Univ. Press, Cambridge, 1990, 349-357

[19] Somer, L., Distribution of residues of certain second-order linear recurrences modulo p. II, Fibonacci Q., 29 (1991), $72-78$

Received: 03.09.2012; In revised form: 28.02.2013; Accepted: 30.03.2013

2010 Mathematics Subject Classification. 11K06, 11B37, 11R06.

Key words and phrases. Distribution modulo 1, Fibonacci sequence. 
[20] Vijayaraghavan, T., On the fractional parts of the powers of a number, J. London Math. Soc., 15 (1940), 159-160

[21] Zhuravleva, V., Diophantine approximations with Fibonacci numbers, J. Théor. Nombres Bordx., 25 (2013), 499-520

DEPARTMENT OF MATHEMATICS AND INFORMATICS

VILNIUS UNIVERSITY

NAUGARDUKO 24, VILNIUS LT-03225, LITHUANIA

E-mail address: arturas.dubickas@mif.vu. It 